\title{
Improving student understanding of addition of angular momentum in quantum mechanics
}

\author{
Guangtian Zhu ${ }^{1,2}$ and Chandralekha Singh ${ }^{2}$ \\ ${ }^{1}$ School of Education Science, East China Normal University, Shanghai, China, 200062 \\ ${ }^{2}$ Department of Physics and Astronomy, University of Pittsburgh, Pittsburgh, Pennsylvania, 15260, USA
}

(Received 12 August 2012; published 7 January 2013)

\begin{abstract}
We describe the difficulties advanced undergraduate and graduate students have with concepts related to addition of angular momentum in quantum mechanics. We also describe the development and implementation of a research-based learning tool, Quantum Interactive Learning Tutorial (QuILT), to reduce these difficulties. The preliminary evaluation shows that the QuILT related to the basics of the addition of angular momentum is helpful in improving students' understanding of these concepts.
\end{abstract}

DOI: 10.1103/PhysRevSTPER.9.010101

PACS numbers: 01.40.Fk

\section{INTRODUCTION}

Quantum mechanics is a particularly challenging subject for undergraduate students. Based upon the research studies that have identified difficulties [1-9], we have developed a set of research-based learning tools to help students develop a good grasp of quantum mechanics [10-14]. These research-based learning tools include the Quantum Interactive Learning Tutorials (QuILTs) and concept tests similar to those popularized by Mazur for introductory physics courses [15]. The QuILTs use a guided inquiry-based approach to learning and help students in building a knowledge structure by guiding them to discern the structure of quantum mechanics. The instructors can use the QuILTs as either in-class tutorials or homework supplements $[12,13]$. The concept tests are integrated with lectures and encourage students to take advantage of their peers' expertise and learn from each other [15].

In this paper, we focus on our investigation to identify student difficulties with concepts related to the addition of angular momentum in quantum mechanics. In the course of this investigation, we found that the main source of difficulties with concepts related to the addition of angular momentum was that the students were not comfortable with the prerequisites, e.g., the basics of a single spin system (dimensionality of a vector space, how to choose a basis and write operators in a given basis, etc.) and the basics of multispin systems (dimensionality of a product space, how to write a complete set of basis vectors in the product space, e.g., in the coupled or uncoupled representation, how to write operators in a given basis, etc.). Then, we developed and assessed a research-based QuILT to help undergraduate students better grasp the preliminary concepts to aid them in understanding the formalism of the addition of angular momentum. The QuILT can also be

Published by the American Physical Society under the terms of the Creative Commons Attribution 3.0 License. Further distribution of this work must maintain attribution to the author(s) and the published article's title, journal citation, and DOI. used by underprepared graduate students as a self-study tool. The investigation of students' difficulties with the addition of angular momentum was conducted with the undergraduate and graduate students at the University of Pittsburgh and other universities [16-19] by administering written tests and conducting in-depth individual interviews with a subset of them.

The research-based QuILT relating to the prerequisites for the addition of angular momentum was administered to students in the second semester of a full-year junior-senior level quantum mechanics course. It strives to build on students' prior knowledge, actively engaging them in the learning process and helping them build connections between the abstract formalism and conceptual aspects of quantum physics, without compromising the technical content. To assess the effectiveness of the QuILT, a pre-test and a post-test related to the addition of angular momentum were given to two classes of undergraduate students at the University of Pittsburgh. We will discuss the results and findings in a later section.

\section{BACKGROUND}

Classically, the angular momentum vector $\vec{L}$ is defined by the cross product of the position $\vec{r}$ and momentum $\vec{p}$; i.e., $\vec{L}=\vec{r} \times \vec{p}$. In quantum mechanics, in which for every observable there is an operator, the components of the orbital angular momentum operator, $\hat{L}_{x}, \hat{L}_{y}$, and $\hat{L}_{z}$, do not commute with each other $\left(\left[\hat{L}_{x}, \hat{L}_{y}\right]=i \hbar \hat{L}_{z},\left[\hat{L}_{y}, \hat{L}_{z}\right]=\right.$ $i \hbar \hat{L}_{x},\left[\hat{L}_{z}, \hat{L}_{x}\right]=i \hbar \hat{L}_{y}$ ), and therefore the components of orbital angular momentum are mutually incompatible observables. The eigenvalues of the square of the magnitude of the orbital angular momentum operator $\hat{L}^{2}$ are $\ell(\ell+1) \hbar^{2}$, where $\ell$, the orbital angular momentum quantum number, is a non-negative integer and $\hbar \equiv h /(2 \pi)$ is the reduced Planck's constant. The eigenvalues of $\hat{L}_{z}$ are $m \hbar$, where $m=-\ell,-\ell+1, \ldots, \ell$. Since $\hat{L}^{2}$ and $\hat{L}_{z}$ commute $\left(\left[\hat{L}^{2}, \hat{L}_{z}\right]=0\right)$, we can use the orbital angular momentum quantum numbers $\ell$ and $m$ to denote their simultaneous eigenstates as $|\ell, m\rangle$. 
In addition to the orbital angular momentum $\vec{L}$, elementary particles, such as electrons, also possess intrinsic spin angular momentum $\vec{S}$ (or spin for short), which is not due to motion in position space. The algebras of the orbital and spin angular momenta are similar and the components of the spin angular momentum operator, $\hat{S}_{x}, \hat{S}_{y}$, and $\hat{S}_{z}$, satisfy commutation relations similar to the commutation relations among the components of the orbital angular momentum operator, $\hat{L}_{x}, \hat{L}_{y}$ and $\hat{L}_{z}$; i.e., $\left[\hat{S}_{x}, \hat{S}_{y}\right]=i \hbar \hat{S}_{z}$, $\left[\hat{S}_{y}, \hat{S}_{z}\right]=i \hbar \hat{S}_{x},\left[\hat{S}_{z}, \hat{S}_{x}\right]=i \hbar \hat{S}_{y},\left[\hat{S}^{2}, \hat{S}_{z}\right]=0$. The eigenvalues of the square of the magnitude of the spin angular momentum operator $\hat{S}^{2}$, are $s(s+1) \hbar^{2}$, where $s$ is the spin quantum number. The spin quantum number $s$ can be a non-negative integer or a non-negative half-odd integer. For the electron, the spin quantum number $s$ is $1 / 2$ and the values of $m_{s}$, the spin quantum number for the $z$ component of spin, are $\pm 1 / 2$. If we choose the eigenstates of the $z$ component of spin as the basis vectors, the operators, $\hat{S}_{x}=$ $\frac{\hbar}{2} \hat{\sigma}_{x}, \hat{S}_{y}=\frac{\hbar}{2} \hat{\sigma}_{y}$, and $\hat{S}_{z}=\frac{\hbar}{2} \hat{\sigma}_{z}$, can be represented by the Pauli matrices,

$$
\begin{gathered}
\hat{\sigma}_{x}=\left(\begin{array}{ll}
0 & 1 \\
1 & 0
\end{array}\right), \quad \hat{\sigma}_{y}=\left(\begin{array}{cc}
0 & -i \\
i & 0
\end{array}\right), \\
\text { and } \quad \hat{\sigma}_{z}=\left(\begin{array}{cc}
1 & 0 \\
0 & -1
\end{array}\right),
\end{gathered}
$$

respectively. Because $\hat{S}^{2}$ and $\hat{S}_{z}$ commute, we can use the quantum numbers $s$ and $m_{s}$ to denote their simultaneous eigenstates as $\left|s, m_{s}\right\rangle$.

If a quantum system contains two particles with individual orbital angular momentum quantum numbers $\ell_{1}$ and $\ell_{2}$, the total orbital angular momentum quantum number of the system can range from $\ell_{1}+\ell_{2}$ down to $\left|\ell_{1}-\ell_{2}\right|$; i.e., $\ell=\ell_{1}+\ell_{2}, \ell_{1}+\ell_{2}-1, \ldots,\left|\ell_{1}-\ell_{2}\right|$. The $z$ component of the total orbital angular momentum of the system equals the sum of the $z$ components of the orbital angular momenta of the individual particles; i.e., $m=m_{1}+m_{2}$. For a single particle with nonzero spin, the possible values of its total angular momentum quantum number $j$ can be obtained by the addition of its orbital angular momentum quantum number $\ell$ and its spin angular momentum quantum number $s$ appropriately; i.e., $j=\ell+s, \ell+s-$ $1, \ldots,|\ell-s|$. Similarly, for two particles with total angular momentum quantum numbers $j_{1}$ and $j_{2}$, the total angular momentum quantum number of the system is $j=j_{1}+j_{2}, j_{1}+j_{2}-1, \ldots,\left|j_{1}-j_{2}\right|$.

In the junior-senior level quantum mechanics courses, the QuILT is developed with learning goals to help students develop a better understanding of the following three issues related to addition of angular momentum.

\section{A. Recognizing the dimension of a Hilbert space}

The dimension of a Hilbert space is equal to the number of linearly independent basis vectors, e.g., the number of linearly independent system eigenstates of any operator representing a system observable that acts on the states in that space. For example, for a particle in a one-dimensional (1D) infinite square well, the infinitely many energy eigenstates $\left|\psi_{n}\right\rangle$ of the Hamiltonian operator form a complete set of basis vectors for the infinite-dimensional Hilbert space.

The Hilbert space corresponding to the spin angular momentum of a single spin- $1 / 2$ particle is two dimensional. For example, the $z$ component of the spin of an electron has two eigenstates, $\left|s=1 / 2, m_{s}=1 / 2\right\rangle$ and $\left|s=1 / 2, m_{s}=-1 / 2\right\rangle \quad\left(\right.$ or $\quad\left|m_{s}=1 / 2\right\rangle \quad$ and $\quad \mid m_{s}=$ $-1 / 2\rangle$ for short, since $s=1 / 2$ is a fixed number for an electron). If a system consists of two electrons, the product space corresponding to the spin degrees of freedom will be four dimensional, which is the product of the dimensions of the Hilbert spaces of each of the spins separately. The basis vectors of the four-dimensional product space in the uncoupled representation are $\left|m_{s 1}=1 / 2\right\rangle \otimes\left|m_{s 2}=1 / 2\right\rangle,\left|m_{s 1}=1 / 2\right\rangle \otimes\left|m_{s 2}=-1 / 2\right\rangle$, $\left|m_{s 1}=-1 / 2\right\rangle \otimes\left|m_{s 2}=1 / 2\right\rangle, \quad$ and $\quad\left|m_{s 1}=-1 / 2\right\rangle \otimes$ $\left|m_{s 2}=-1 / 2\right\rangle(\otimes$ is used to denote the direct product or the Kronecker product [20]).

\section{B. Choosing the basis vectors for the Hilbert space}

For a system consisting of two spin- $1 / 2$ particles, there are two common ways to represent the basis vectors for the product space. Since the spin quantum numbers $s_{1}=1 / 2$ and $s_{2}=1 / 2$ are fixed, we can use the "uncoupled representation" and express the orthonormal basis vectors for the product space as $\left|s_{1}, m_{1}\right\rangle \otimes$ $\left|s_{2}, m_{2}\right\rangle=\left|m_{1}\right\rangle \otimes\left|m_{2}\right\rangle$, as noted earlier. In this uncoupled representation, the operators related to each particle (subspace) act on their own states; e.g., $\hat{S}_{1 z}|1 / 2\rangle_{1} \otimes|-1 / 2\rangle_{2}=\frac{\hbar}{2}|1 / 2\rangle_{1} \otimes|-1 / 2\rangle_{2}$ and $\hat{S}_{2 z}|1 / 2\rangle_{1} \otimes$ $|-1 / 2\rangle_{2}=-\frac{\hbar}{2}|1 / 2\rangle_{1} \otimes|-1 / 2\rangle_{2}$. On the other hand, we can use the "coupled representation" and find the total spin quantum number for the system of two particles together. The total spin quantum number for the two spin- $1 / 2$ particle system $s$ is either $1 / 2+1 / 2=1$ or $1 / 2-1 / 2=0$. When the total spin quantum number $s$ is 1 , the quantum number $m_{s}$ for the $z$ component of the total spin $S_{z}$ can be 1,0 , and -1 . When the total spin is $0, m_{s}$ can only be 0 . Therefore, the basis vectors of the system in the coupled representation are $\mid s=$ $\left.1, m_{s}=1\right\rangle,\left|s=1, m_{s}=0\right\rangle,\left|s=1, m_{s}=-1\right\rangle$, and $\mid s=$ $\left.0, m_{s}=0\right\rangle$. In the coupled representation, the state of a two-spin system is not a simple product of the states of each individual spin although we can write each coupled state as a linear superposition of a complete set of uncoupled states. For example, the normalized basis vectors in the coupled representation $\mid s=1, m_{s}=$ $1\rangle,\left|s=1, m_{s}=0\right\rangle,\left|s=1, m_{s}=-1\right\rangle$, and $\mid s=0, m_{s}=$ $0\rangle$ can be expressed in the uncoupled representation as follows: 


$$
\begin{aligned}
& \left|s=1, m_{s}=1\right\rangle=\left|m_{1}=1 / 2\right\rangle \otimes\left|m_{2}=1 / 2\right\rangle, \\
& \left|s=1, m_{s}=0\right\rangle=\left(\left|m_{1}=1 / 2\right\rangle \otimes\left|m_{2}=-1 / 2\right\rangle+\left|m_{1}=-1 / 2\right\rangle \otimes\left|m_{2}=1 / 2\right\rangle\right) / \sqrt{2}, \\
& \left|s=1, m_{s}=-1\right\rangle=\left|m_{1}=-1 / 2\right\rangle \otimes\left|m_{2}=-1 / 2\right\rangle, \\
& \left|s=0, m_{s}=0\right\rangle=\left(\left|m_{1}=1 / 2\right\rangle \otimes\left|m_{2}=-1 / 2\right\rangle-\left|m_{1}=-1 / 2\right\rangle \otimes\left|m_{2}=1 / 2\right\rangle\right) / \sqrt{2} .
\end{aligned}
$$

\section{Constructing the matrix of angular momentum operators}

To calculate the diagonal and off-diagonal matrix elements of an operator in the product space, we must compute the matrix elements of that operator in the appropriate basis. For example, for a two spin-1/2 particle system, for the operator $\hat{S}_{1 z}+\hat{S}_{2 z}$, when we use the basis vectors in the uncoupled representation, the matrix elements are $\left\langle m_{2}^{\prime}\right| \otimes$ $\left\langle m_{1}^{\prime}\left|\hat{S}_{1 z}+\hat{S}_{2 z}\right| m_{1}\right\rangle \otimes\left|m_{2}\right\rangle$, where $m_{1}, m_{2}, m_{1}^{\prime}, m_{2}^{\prime}$ are either $1 / 2$ or $-1 / 2$. If we choose the order of the basis vectors to be $|1 / 2\rangle_{1} \otimes|1 / 2\rangle_{2},|1 / 2\rangle_{1} \otimes|-1 / 2\rangle_{2},|-1 / 2\rangle_{1} \otimes|1 / 2\rangle_{2}$, and $|-1 / 2\rangle_{1} \otimes|-1 / 2\rangle_{2}$, the operator matrix is

$$
\hat{S}_{1 z}+\hat{S}_{2 z}=\left(\begin{array}{cccc}
\hbar & 0 & 0 & 0 \\
0 & 0 & 0 & 0 \\
0 & 0 & 0 & 0 \\
0 & 0 & 0 & -\hbar
\end{array}\right) \text {. }
$$

The basis vectors in the coupled representation $\left|s, m_{s}\right\rangle$ are also a good choice to express this operator in matrix form, and the matrix is diagonal since $\left|s, m_{s}\right\rangle$ are the eigenstates of the $z$ component of the total spin operator $\hat{S}_{z}=\hat{S}_{1 z}+\hat{S}_{2 z}$. When we construct the operator matrix by using the basis vectors for the coupled representation in the order $|1,1\rangle,|1,0\rangle,|1,-1\rangle$, and $|0,0\rangle$, the operator matrix can be expressed as

$$
\hat{S}_{1 z}+\hat{S}_{2 z}=\left(\begin{array}{cccc}
\hbar & 0 & 0 & 0 \\
0 & 0 & 0 & 0 \\
0 & 0 & -\hbar & 0 \\
0 & 0 & 0 & 0
\end{array}\right) .
$$

We can also rearrange the sequence of the basis as $|1,1\rangle$, $|1,-1\rangle,|1,0\rangle$, and $|0,0\rangle$ in order to move the nonzero matrix elements, e.g., to the upper left corner. Although the matrices of the particular operator $\hat{S}_{z}=\hat{S}_{1 z}+\hat{S}_{2 z}$ are diagonal in both the coupled and uncoupled representations, in general, the operator matrices are different in different basis sets (an issue discussed at length in the QuILT). In particular, the matrix corresponding to an operator may be diagonal in one representation but not in another representation.

\section{INVESTIGATION OF STUDENTS' DIFFICULTIES}

The investigation of difficulties was carried out by administering free-response and multiple-choice questions to advanced undergraduate students enrolled in quantum mechanics courses. Individual interviews were also carried out, to better understand students' rationale for their responses, before, during, and after the development of different versions of the QuILT on the preliminaries of the addition of angular momentum and the corresponding pre-test and post-test. In addition to informal discussions with students, we conducted one-on-one interviews (each lasting between 1 and 2 hours) with 15 undergraduate and graduate students using a think-aloud protocol. In the interviews, students were asked to articulate their reasoning processes while they answered the questions. Students were not prompted unless they remained silent for a while. At the end of the interviews, students were asked to clarify the issues they had not made clear earlier. After each individual interview on a particular version of the QuILT (along with the pre-test and the post-test administered), modifications were made based upon the feedback obtained from students' performance on the QuILT, the pre-test, and the post-test. For example, if students got stuck at a particular point and could not make progress from one question to the next, modifications were made accordingly.

\section{A. Difficulty with the dimension of a Hilbert space}

Difficulty A.1: Confusion between Hilbert space and position space even before learning about addition of angular momentum.

We have found that the concepts related to Hilbert space are very difficult for students, and many students were confused about the dimensions of the Hilbert space and the position space. The following multiple-choice question was given to 33 undergraduate students (before instruction in addition of angular momentum, but after instruction in relevant concepts) to probe whether they could distinguish between the one-dimensional position space in which the particle is confined and the infinite-dimensional Hilbert space of system states.

- Choose all of the following statements that are correct for a particle interacting with a one dimensional (1D) infinite square well.

(1) The appropriate Hilbert space for this system is one dimensional.

(2) The energy eigenstates of the system form a basis in a $1 D$ Hilbert space. 
(3) The position eigenstates of the system form a basis in a $1 D$ Hilbert space.

A. none of the above B. 1 only C. 2 only D. 3 only E. all of the above

The Hilbert space in which the state of the system lies is infinite dimensional, while the position space in which the particle is confined is one dimensional. However, only $48 \%$ of the students chose the correct answer A. About 25\% of the students selected the answer $\mathrm{E}$ and incorrectly believed that both the energy eigenstates and position eigenstates form a basis in a 1D Hilbert space. This example illustrates that the students have difficulties with the dimension of the Hilbert space even before addition of angular momentum is discussed in the quantum mechanics course. For a given quantum system, the translational and spin degrees of freedom are distinct. For example, the vector space corresponding to the spin angular momentum or the orbital angular momentum of an atom is finite dimensional, while the vector space of the translational degrees of freedom is infinite dimensional. Therefore, when discussing the angular momentum of quantum particles, the translational degrees of freedom of the system were not emphasized in the QuILT in order to maintain students' focus on addition of angular momentum and avoid further confusions about the dimension of Hilbert space.

Difficulty A.2: Incorrectly calculating the dimension of a product space by adding the dimensions of the subspaces.

Students in general have great difficulty finding the dimension of a product space containing two or more angular momenta. When asked about the dimension $D$ of a product space consisting of two subspaces of dimensions $D_{1}$ and $D_{2}$, many students incorrectly believed that $D=$ $D_{1}+D_{2}$ instead of $D_{1} \times D_{2}$. Discussions with individual students suggest that such a misconception often originates from two reasons. One reason is the word "addition" in "addition of angular momentum." The second reason is related to the simplest example in which students learn about the product space for two spin- $1 / 2$ particles. In this case, the dimension of the product space is four, which equals $2 \times 2$ but is also $2+2$. When we asked 11 students about the dimension of the product space for a system containing two spin-1 particles, 4 of them provided the incorrect answer $6=3+3$ instead of the correct answer $9=3 \times 3$. Individual discussions with students also suggest that students are confused about the dimension of the product space. It appears, therefore, that using the example of two two-dimensional spaces is a poor choice unless students are also given ample opportunity to contemplate the results for other product spaces (which is done in the QuILT). Because of its simplicity, the product space for two two-dimensional spaces is the choice commonly used to illustrate issues related to the addition of angular momentum, and students may or may not have the opportunity to extend these results to other cases.

\section{B. Difficulty in identifying different basis vectors for the product space}

Difficulty B.1: Difficulty in choosing a convenient basis to represent an operator as an $N \times N$ matrix in an $N$-dimensional product space.

Students often have difficulty in figuring out when it would be convenient to choose the basis vectors for the product space to be in the coupled or uncoupled representations, and many have difficulty in writing an operator in a matrix form in the chosen basis. For example, when 26 students were asked to choose a basis for two spin-1/2 particles and write down the matrix corresponding to the operator $\hat{S}_{1} \cdot \hat{S}_{2}=\left(\hat{S}^{2}-\hat{S}_{1}^{2}-\hat{S}_{2}^{2}\right) / 2$ in that basis, $15 \%$ of the students could not find a complete set of basis vectors for the product space. Moreover, those who chose the uncoupled representation often had difficulty figuring out how to write $\hat{S}^{2}$ in a matrix form even though they were given the appropriate Clebsch-Gordon coefficient (CGC) table to write the coupled states in terms of uncoupled states and vice versa. About $33 \%$ of the students did not realize that the basis vectors in the coupled representation are eigenstates of the operator $\hat{S}^{2}$, so that the matrix elements of $\hat{S}_{1} \cdot \hat{S}_{2}$ can be calculated more easily in the coupled representation than in the uncoupled representation. Some students mistakenly thought that the basis vectors in the product space are simply a collection of the basis vectors for the subspaces. For example, for the two spin-1/2 particle system, about $8 \%$ of the students incorrectly wrote down the basis vectors as $\left|s_{1}=1 / 2, m_{1}=1 / 2\right\rangle, \mid s_{1}=1 / 2, m_{1}=$ $-1 / 2\rangle,\left|s_{2}=1 / 2, m_{2}=1 / 2\right\rangle$, and $\left|s_{2}=1 / 2, m_{2}=-1 / 2\right\rangle$ and constructed incorrect $2 \times 2$ matrices for the operators they were asked to write in the matrix form in their chosen basis.

Difficulty B.2: Incorrectly believing that if the operator matrix is diagonal in one representation, it must also be diagonal in another representation.

To evaluate students' understanding of operators in coupled and uncoupled representations, the following multiple-choice question was given to 25 students.

- Suppose the Hamiltonian of a two spin-1/2 particle system is $\hat{H}=\gamma\left(\hat{\vec{S}}_{1} \cdot \vec{B}_{1}+\hat{\vec{S}}_{2} \cdot \vec{B}_{2}\right)$ in which the magnetic fields $\vec{B}_{1}$ and $\vec{B}_{2}$ are both in the $z$-direction but with different magnitudes and $\gamma$ is a suitable constant. Choose all of the following statements that are correct.

(1) The Hamiltonian is a diagonal matrix in the coupled representation $\left|S^{2}, S_{1}^{2}, S_{2}^{2}, S_{z}\right\rangle$.

(2) The Hamiltonian is a diagonal matrix in the uncoupled representation $\left|S_{1}^{2}, S_{1 z}\right\rangle \otimes\left|S_{2}^{2}, S_{2 z}\right\rangle$.

(3) The Hamiltonian is a $2 \times 2$ matrix in the uncoupled representation 


$$
\hat{H}=\frac{\gamma \hbar B_{1}}{2}\left(\begin{array}{cc}
1 & 0 \\
0 & -1
\end{array}\right)+\frac{\gamma \hbar B_{2}}{2}\left(\begin{array}{cc}
1 & 0 \\
0 & -1
\end{array}\right) .
$$

Since the basis vectors in the coupled representation, $\left|S^{2}, S_{1}^{2}, S_{2}^{2}, S_{z}\right\rangle$, are not the eigenstates of the Hamiltonian, some off-diagonal elements of $\hat{H}$ will be nonzero. These off-diagonal matrix elements can be calculated by writing the coupled states in terms of uncoupled states. However, when the $\hat{H}$ matrix is expressed in the uncoupled representation, all of the off-diagonal elements are zero since the basis vectors $\left|S_{1}^{2}, S_{1 z}\right\rangle \otimes\left|S_{2}^{2}, S_{2 z}\right\rangle$ are the orthogonal eigenstates of $\hat{H}$. While the correct answer is (2) only, about $40 \%$ of the students chose both the options (1) and (2). Some students incorrectly believed that the Hamiltonian must be diagonal in both the coupled and uncoupled representations. In individual discussions, students were asked to write the operator $\hat{S}_{1 z}+\frac{1}{2} \hat{S}_{2 z}$ for a two spin- $1 / 2$ particle system in matrix form in the product space. During individual discussions, a student incorrectly believed that $\hat{S}_{1 z}+\frac{1}{2} \hat{S}_{2 z}$ is diagonal in the coupled representation. When he was told that the matrix was not diagonal in the coupled representation, he claimed “. $\ldots \hat{S}_{1 z}+\hat{S}_{2 z}$ is a diagonal matrix in the coupled basis. How can there be any difference between that operator and the operator $\hat{S}_{1 z}+\frac{1}{2} \hat{S}_{2 z}$ when it is also a linear combination of $\hat{S}_{1 z}$ and $\hat{S}_{2 z}$ ?" The student had failed to observe that $\hat{S}_{z}=\hat{S}_{1 z}+\hat{S}_{2 z}$ is a very special linear combination of $\hat{S}_{1 z}$ and $\hat{S}_{2 z}$ which is diagonal in both the coupled and uncoupled representations but other linear combinations of $\hat{S}_{1 z}$ and $\hat{S}_{2 z}$ will not be diagonal in the coupled representation.

\section{Difficulty in constructing an operator matrix in the product space and realizing that the matrix corresponding to an operator could be very different in a different basis}

As described in Sec. II, the matrix corresponding to an operator will depend on the basis chosen. However, many students had difficulty realizing that the matrix of the same operator can be very different for a different basis. Below, we describe some related difficulties in more depth.

Difficulty C.1: Mistakenly adding the operators in different Hilbert spaces algebraically to construct the operator for the product space as if they act in the same Hilbert space.

We find that the students often have difficulty in constructing matrix representations of operators correctly in the product space. For example, when 26 students were asked to construct the matrix of $\hat{S}_{1 z}+\hat{S}_{2 z}$ in a suitable basis, about $1 / 4$ of them incorrectly claimed that the resulting matrix is two dimensional and they simply added up the matrices of the operators $\hat{S}_{1 z}$ and $\hat{S}_{2 z}$, i.e.,

$$
\hat{S}_{1 z}+\hat{S}_{2 z}=\frac{\hbar}{2}\left(\begin{array}{cc}
1 & 0 \\
0 & -1
\end{array}\right)_{1}+\frac{\hbar}{2}\left(\begin{array}{cc}
1 & 0 \\
0 & -1
\end{array}\right)_{2}
$$

Some of these students placed subscripts 1 and 2 on the matrices to differentiate the two spin- $1 / 2$ particles, but most just merged them into a single matrix. Similar difficulties were found when these students were asked to construct a matrix for the operator $\hat{S}_{1} \cdot \hat{S}_{2}$ in any suitable basis of their choice. For example, three out of the 26 students simply multiplied the $2 \times 2$ matrices corresponding to each of the spins and expressed the result as another $2 \times 2$ matrix. Also, more than half of the students had difficulty realizing that the operator $\hat{S}_{1 z}$ in the product space of two spin- $1 / 2$ systems is a $4 \times 4$ matrix (and not a $2 \times 2$ matrix).

Difficulty C.2: Incorrectly believing that the dimension of the operator matrix depends on the choice of basis vectors.

The dimension of the product space is independent of the basis or representation chosen. For example, both the uncoupled and coupled representations for the two spin-1/2 particle system have four basis vectors since the product space is four dimensional. Several students displayed an inconsistency in interpreting the dimension of the product space depending upon the basis chosen. For example, 5 out of 11 students incorrectly believed that the matrix for the operator $\hat{S}_{1 z}+\hat{S}_{2 z}$ is two dimensional in the uncoupled representation. However, when explicitly asked about the same operator in the coupled representation, they could correctly construct a $4 \times 4$ diagonal matrix with the eigenvalues of $\hat{S}_{z}=\hat{S}_{1 z}+\hat{S}_{2 z}$ in the diagonal positions using the basis vectors $|1,1\rangle,|1,-1\rangle,|1,0\rangle$, and $|0,0\rangle$. Discussions with individual students suggest that some of them may know that the operator matrices are in general different in different basis sets, but they were unclear about the fact that the dimension of the product space should always be equal to the number of linearly independent vectors in that space and it cannot depend on the choice of basis vectors.

Difficulty C.3: The Hamiltonian of the system must be known in order to construct a matrix for an operator other than the Hamiltonian operator.

We asked the students to construct the matrix for the operator $\hat{S}_{1 z}+\frac{1}{2} \hat{S}_{2 z}$ of a two spin-1/2 particle system with only spin degrees of freedom involved. We found that some students believed that the Hamiltonian of the system must be given in order for them to be able to find the matrix elements of other operators. Discussions with individual students suggest that this misconception originates from several sources. First, some students believed that since the basis vectors are often selected to be the eigenstates of the Hamiltonian, these are the only basis vectors that can be used to construct the matrix for any operator. Also, students were taught how to construct the Hamiltonian matrix for a single electron spin in a uniform magnetic 
field (Larmor precession of spin) and later they were taught how to construct the matrix of a Hamiltonian such as $\hat{H}=$ $\gamma\left(\hat{S}_{1} \cdot \vec{B}+\hat{S}_{2} \cdot \vec{B}\right)$ in the product space. There is also emphasis throughout the course on the role of the Hamiltonian in determining the time evolution of the system and allowed energies. Thus, some of the students overgeneralized the importance of the Hamiltonian in other contexts and claimed that they cannot construct the matrix for the operator $\hat{S}_{1 z}+\frac{1}{2} \hat{S}_{2 z}$ in the product space without knowing the Hamiltonian of the system.

\section{Difficulty in finding the probabilities for measuring an observable in a product space}

A particular choice of basis vectors for the product space may be suitable for answering questions related to the probabilities of measuring a particular observable. For example, if the question is related to the probabilities of measuring the observable $S_{1 z}$ or $S_{2 z}$ in a product state written in the coupled representation, it is convenient to go to the uncoupled representation. Similarly, writing the states in the product space in the coupled basis may be convenient for other probabilities, e.g., the probability of measuring the square of the magnitude of the total spin angular momentum $\vec{S}^{2}$.

Students had difficulties in choosing a convenient basis in the product space for answering questions related to probabilities of measuring a particular observable. This difficulty was partly due to the fact that students did not realize which basis vectors were eigenvectors of the operator corresponding to a particular observable and that it is easy to find the probabilities of measuring an observable if the state of the system is written in terms of the eigenstates of that observable. Another type of difficulty was related to transforming from one basis to another (e.g., from coupled to uncoupled or vice versa) using the CGC table provided and collecting all of the coefficients of a given state before taking the absolute square of a coefficient to find the probability of measuring an observable which has a definite value in that state. This latter difficulty in collecting the coefficients of a particular state before taking the absolute square to find the probability in product space is similar to those observed for a single spin. It can be illustrated with the following example. Suppose a single spin- $1 / 2$ state is given by the following expression $\left(\left|s, m_{s}\right\rangle=|1 / 2, \pm 1 / 2\rangle\right)$ after certain manipulations:

$|\psi\rangle=\left(a\left|\frac{1}{2}, \frac{1}{2}\right\rangle+a^{\prime}\left|\frac{1}{2},-\frac{1}{2}\right\rangle\right)+\left(b\left|\frac{1}{2}, \frac{1}{2}\right\rangle+b^{\prime}\left|\frac{1}{2},-\frac{1}{2}\right\rangle\right)$,

where $a, a^{\prime}, b$, and $b^{\prime}$ are constant coefficients. When we asked 17 students to calculate the probability of obtaining $\hbar / 2$ for measuring the observable $S_{z}$ (corresponding to $\left.m_{s}=1 / 2\right), 12 \%$ of the students incorrectly responded that it was $|a|^{2}+|b|^{2}$. However, the coefficients with the same basis vector $\left|s=1 / 2, m_{s}=1 / 2\right\rangle$ should be combined first as $(a+b)\left|s=1 / 2, m_{s}=1 / 2\right\rangle$ to yield the correct probability for obtaining $\hbar / 2$ to be $|a+b|^{2}$. This difficulty related to finding the appropriate probability amplitude by combining the coefficients of the same basis vectors persisted when dealing with a product space and using the CGC table to transform from one basis to another. Such difficulty may also partly be related to the general difficulties students have in expressing quantities in the simplest algebraic form.

\section{E. Difficulty in dealing with the product space corresponding to the addition of orbital angular momentum and spin angular momentum of a single particle.}

Some students thought that the addition of angular momentum formalism only applies to a quantum system containing two or more particles (e.g., for adding the spin angular momentum of different particles). They did not realize that the orbital angular momentum and the spin angular momentum of a single particle also follow the same rule of addition of angular momentum. For example, since the orbital angular momentum state $|\ell, m\rangle$ can be expressed in the form of a spherical harmonic function $Y_{\ell}^{m}$, the total angular momentum part (including both spin and orbital degrees of freedom but excluding the radial part) of a specific state of a single spin- $1 / 2$ particle with $\ell=1$ orbital angular momentum quantum number can be written as, e.g., $\quad \sqrt{2 / 3} Y_{1}^{0}\left|s=1 / 2, m_{s}=1 / 2\right\rangle+\sqrt{1 / 3} Y_{1}^{1} \mid s=$ $\left.1 / 2, m_{s}=-1 / 2\right\rangle$ in the uncoupled representation. If we use the CGC table to transform the basis vectors from the uncoupled representation to the coupled representation, the same state can be written as $\left|j=3 / 2, m_{j}=1 / 2\right\rangle$, where $j$ is the quantum number for the total angular momentum and $m_{j}$ is the quantum number for the $z$ component of the total angular momentum. However, when we asked students to find the outcome and the corresponding probability if they measure the $z$ component of the total angular momentum of a particle in the state $\sqrt{2 / 3} Y_{1}^{0}\left|s=1 / 2, m_{s}=1 / 2\right\rangle+$ $\sqrt{1 / 3} Y_{1}^{1}\left|s=1 / 2, m_{s}=-1 / 2\right\rangle$, only 5 out of 11 students answered this question correctly. Many students either forgot that the spherical harmonic function $Y_{\ell}^{m}$ corresponds to the orbital angular momentum state $|\ell, m\rangle$ or mistakenly thought that $Y_{1}^{0}$ and $Y_{1}^{1}$ represent the spin states $\mid s=$ $\left.1 / 2, m_{s}= \pm 1 / 2\right\rangle$. Interviews with the students also indicated that some students have difficulties in differentiating the related concepts about the orbital angular momentum and the total angular momentum of a single particle. Some interviewed students were confused and were surprised to know that the addition of angular momentum formalism is applicable regardless of whether we add orbital and spin angular momenta, two orbital angular momenta, or two spin angular momenta.

\section{IMPROVING STUDENTS' UNDERSTANDING WITH THE QUILT}

We have developed a QuILT to help reduce the difficulties faced by students in learning about the preliminaries of 
angular momentum addition. The QuILT focuses on the preliminaries, e.g., the dimensionality of the product space, how to choose a complete set of basis vectors for the product space, and how to use these basis vectors to calculate the matrix elements for an operator and construct an operator matrix. The QuILT focuses on helping students learn about the coupled and uncoupled representations, but does not teach them about going from one representation to another. This is because during the investigation of difficulties we found that most students were struggling with the preliminaries and those who learned the preliminaries were able to comprehend the treatment in the common textbooks about how to change basis using CGC tables. The QuILT builds on the prior knowledge of students as determined by our investigation of difficulties. The concepts covered in the QuILT are gradually built up in a guided inquiry-based approach [21]. The QuILT was developed based on the difficulties found by written surveys and interviews. The development of the QuILT went through a cyclical interactive process which included the following stages: (1) development of the preliminary version based on a theoretical analysis of the underlying knowledge structure and research on student difficulties, (2) implementation and evaluation of the QuILT by administering it individually to students, (3) determining its impact on student learning and assessing what difficulties remained, and (4) refinements and modifications based on the feedback from the implementation and evaluation.

As noted earlier, the QuILT on the addition of angular momentum helps with the issues related to finding the dimensionality of the product space and a complete set of basis vectors in each of the coupled and uncoupled representations. The QuILT also strives to help the students learn how to use these basis vectors to construct any operator matrix in the coupled or uncoupled representation by calculating the matrix elements (sandwiching the operator between the state vectors). From the QuILT, students can learn that an operator matrix may not be the same in the coupled and uncoupled representations; regardless, the dimensionality of a product space in coupled and uncoupled representations is always the same. Students are given an opportunity to generalize all of the above issues from the case of two spin-1/2 particles (four-dimensional product space) to other cases with higher dimensions. However, the QuILT does not explicitly teach the students how to write the basis vectors in coupled representations in terms of the basis vectors in uncoupled representations or how to convert a matrix in the coupled representation to a matrix in the uncoupled representation. As noted earlier, many students were able to follow the treatment in the standard textbooks to accomplish such tasks once they had worked on the QuILT to learn the preliminaries. Such topics may be included in future supplementary tutorials. In the current version of the QuILT, we focus on foundational topics with which students had great difficulties, including the dimensionality of a product space, choosing a complete set of basis vectors for the product space, the differences between the coupled and uncoupled representations, constructing any operator matrix in a given basis, etc.

After iterating different versions of the QuILT with individual students until the post-test performance of individual students who worked on the QuILT significantly improved compared to the pre-test performance, it was administered in undergraduate quantum mechanics classes after traditional instruction on the addition of angular momentum. On average, students spend around 1.5 hours on the QuILT on the addition of angular momentum, which contains many questions (most of which are in the multiple-choice format to ensure that the QuILT can eventually be turned into a Web-based tutorial which students can use as a self-study tool and obtain appropriate feedback if they click on an incorrect option for a multiple-choice question).

\section{A. Dimension of Hilbert space}

Before the QuILT on the basics related to the addition of angular momentum, students are asked to work on a warm-up part which helps them with the basics of a single spin system. Then, students work on the QuILT related to the addition of angular momentum which has two parts, one part related to the uncoupled representation and the other to the coupled representation. At the beginning of the first part of the QuILT, students are asked about the dimension of the product space for the two spin- $1 / 2$ particle system. Together with the correct answer that the dimension is $4=2 \times 2$, a distracter answer $4=2+2$ was also given in the multiple-choice question. This strategy forces students to notice the difference between these two answers and learn about the product space dimension by discussing their answers with their peers. Note that the QuILT can be used as a self-study tool, but when students work on it in class, peer discussion is exploited throughout. Then the students go through a guided approach to construct the basis vectors in the uncoupled representation for two spin-1/2 particles (each with the $z$ component of spin quantum numbers $\pm 1 / 2)$, e.g., $|1 / 2\rangle_{1} \otimes|-1 / 2\rangle_{2}$ or $|\uparrow\rangle_{1} \otimes|\downarrow\rangle_{2}$, and learn about the fact that the operators $\hat{S}_{1 z}$ and $\hat{S}_{2 z}$ only act on their respective subspaces in the uncoupled representation. After this help in constructing basic understanding of the uncoupled representation, students are asked the following multiple-choice question.

- Choose all the statements that are correct.

$$
\begin{aligned}
& (1)|1 / 2\rangle_{1} \otimes|1 / 2\rangle_{2} \text { is an eigenstate of } \hat{S}_{1 z} \text { and } \hat{S}_{2 z} \text { but not } \\
& \hat{S}_{1}^{2} \text { or } \hat{S}_{2}^{2} \text {. } \\
& \text { (2) }|1 / 2\rangle_{1} \otimes|1 / 2\rangle_{2} \text { is an eigenstate of } \hat{S}_{1 z}, \hat{S}_{2 z}, \hat{S}_{1}^{2} \\
& \text { and } \hat{S}_{2}^{2} \text {. }
\end{aligned}
$$


(3) $|1 / 2\rangle_{1} \otimes|1 / 2\rangle_{2}$ is an eigenstate of $\hat{S}_{1 z}, \hat{S}_{2 z}, \hat{S}_{1}$ and $\hat{S}_{2}$.

Students discuss their answers with peers [correct answer is (2) only] and learn that, in the uncoupled representation, the basis vectors are eigenstates of the individual spin operators $\hat{S}_{1 z}, \hat{S}_{2 z}, \hat{S}_{1}^{2}$, and $\hat{S}_{2}^{2}$. They also learn to calculate the individual matrix elements and construct various matrices in the uncoupled representation (in the first part of the QuILT). For example, students learn that $\left(\hat{S}_{1 z}+\right.$ $\left.\hat{S}_{2 z}\right)|1 / 2\rangle_{1} \otimes|-1 / 2\rangle_{2}=(\hbar / 2-\hbar / 2)|1 / 2\rangle_{1} \otimes|-1 / 2\rangle_{2}=0$. For the operator $\hat{S}_{1 z}+\hat{S}_{2 z}$, they are guided to conclude that one of the matrix elements is ${ }_{2}\langle-1 / 2| \otimes_{1}\langle 1 / 2|\left(\hat{S}_{1 z}+\right.$ $\left.\hat{S}_{2 z}\right)|1 / 2\rangle_{1} \otimes|-1 / 2\rangle_{2}=0$, and they also practice how to find other matrix elements.

In order to generalize their understanding of the product space to more complicated situations, students are later asked to consider the product space of a three spin- $1 / 2$ particle system in the uncoupled basis. One question explicitly asks them to consider the dimension in this case as follows.

- What is the dimensionality of the spin space of a three spin- $1 / 2$ system?

(a) 2 (b) $2+2+2=6$ (c) $3^{2}=9$ (d) $2^{3}=8$

Here students are given an opportunity to think about and discuss with peers the fact that the dimension of a product space is the product of the dimensions of the subspaces [and hence the correct answer is (d)]. They are further asked to construct a complete set of eight basis vectors and then calculate several diagonal and off-diagonal matrix elements of the operator $\hat{S}_{1 z}+\hat{S}_{2 z}+\hat{S}_{3 z}$ in the uncoupled representation.

\section{B. Constructing matrices for different operators for the product space of two spin-1/2 systems in the uncoupled representation}

In the QuILT, students are asked to calculate the matrices of the following operators in the uncoupled representation given that a uniform magnetic field $\vec{B}$ is pointing in the $+z$ direction: $\hat{H}_{1}=\left(4 E_{0} / \hbar\right)\left(\hat{\vec{S}}_{1} \cdot \hat{\vec{S}}_{2}\right)$ and $\hat{H}_{2}=$ $-\mu\left(\hat{\vec{S}}_{1} \cdot \vec{B}+\hat{\vec{S}}_{2} \cdot \vec{B}\right)$. They are told that $\mu$ and $4 E_{0} / \hbar$ are constants. Students must contemplate the properties of the operators $\hat{S}_{1 z}+\hat{S}_{2 z}$ and $\hat{\vec{S}}_{1} \cdot \hat{\vec{S}}_{2}$. Since the basis vectors in the uncoupled representation are orthonormal eigenstates of the operators $\hat{S}_{1 z}$ and $\hat{S}_{2 z}$, all the off-diagonal matrix elements of the operator $\hat{S}_{1 z}+\hat{S}_{2 z}$ are zero. After helping students learn about how to construct the matrix for the operator $\hat{S}_{1 z}+\hat{S}_{2 z}$, students learn how to construct the matrix for a more complicated operator, $\hat{\vec{S}}_{1} \cdot \hat{\vec{S}}_{2}$, in the uncoupled representation. They are first asked the following question to help them think about why it is more convenient to write the operator in the form $\hat{S}_{1 x} \hat{S}_{2 x}+$ $\hat{S}_{1 y} \hat{S}_{2 y}+\hat{S}_{1 z} \hat{S}_{2 z}$ rather than $\hat{S}^{2}-\hat{S}_{1}^{2}-\hat{S}_{2}^{2}$ to calculate the matrix elements in the uncoupled basis (without access to the CGC table).

- Consider the following conversation between Pria and Mira:

Pria: Is $\left(4 E_{0} / \hbar\right) \hat{\vec{S}}_{1} \cdot \hat{\vec{S}}_{2}$ or $\left(2 E_{0} / \hbar\right)\left(\hat{S}^{2}-\hat{S}_{1}^{2}-\hat{S}_{2}^{2}\right)$ the more convenient form for writing $\hat{H}_{1}$ in matrix form in the uncoupled representation?

Mira: Since the basis vectors $\left|m_{s}\right\rangle_{1} \otimes\left|m_{s}\right\rangle_{2}$ are not the eigenstates of $\hat{\vec{S}}_{1} \cdot \hat{\vec{S}}_{2}$ or $\hat{S}^{2}-\hat{S}_{1}^{2}-\hat{S}_{2}^{2}$, we have to be careful. It is the form $\left(4 E_{0} / \hbar\right) \hat{\vec{S}}_{1} \cdot \hat{\vec{S}}_{2}$ that is more useful because we can write $\hat{\vec{S}}_{1} \cdot \hat{\vec{S}}_{2}=\hat{S}_{1 x} \hat{S}_{2 x}+\hat{S}_{1 y} \hat{S}_{2 y}+$ $\hat{S}_{1 z} \hat{S}_{2 z}$. Then we can write the $x$ and $y$ components of spin in terms of the raising and lowering operators and we know how they act on $\left|m_{s}\right\rangle_{1} \otimes\left|m_{s}\right\rangle_{2}$.

Do you agree with Mira? Explain.

The students learn to rewrite the operator $\hat{\vec{S}}_{1} \cdot \hat{\vec{S}}_{2}$ using the raising and lowering operators such that $\hat{\vec{S}}_{1} \cdot \hat{\vec{S}}_{2}=$ $\left(\hat{S}_{1-} \hat{S}_{2+}+\hat{S}_{1+} \hat{S}_{2-}\right) / 2+\hat{S}_{1 z} \hat{S}_{2 z}$ and they practice applying the raising and lowering operators to the basis vectors in the uncoupled representation as in the following example [with correct answer (1)].

- Which one of the following equations is correct?

(1) $\hat{S}_{1-} \hat{S}_{2+}|1 / 2\rangle_{1} \otimes|1 / 2\rangle_{2}=\left(\hat{S}_{1-}|1 / 2\rangle_{1}\right) \otimes\left(\hat{S}_{2+}|1 / 2\rangle_{2}\right)$ $=0$

(2) $\hat{S}_{1-} \hat{S}_{2+}|1 / 2\rangle_{1} \otimes|1 / 2\rangle_{2}=\left(\hat{S}_{1-}|1 / 2\rangle_{1}\right) \otimes\left(\hat{S}_{2+}|1 / 2\rangle_{2}\right)$ $=\hbar^{2}|1 / 2\rangle_{1} \otimes|1 / 2\rangle_{2}$

(3) $\hat{S}_{1-} \hat{S}_{2+}|1 / 2\rangle_{1} \otimes|1 / 2\rangle_{2}=\left(\hat{S}_{1-}|1 / 2\rangle_{1}\right) \otimes\left(\hat{S}_{2+}|1 / 2\rangle_{2}\right)=$ $2 \hbar^{2}|1 / 2\rangle_{1} \otimes|1 / 2\rangle_{2}$

Students learn that calculating the matrix elements of an operator in the uncoupled basis can be achieved by expressing the operator as a combination of $\hat{S}_{1 z}, \hat{S}_{2 z}, \hat{S}_{1 \pm}$, and $\hat{S}_{2 \pm}$ since operators corresponding to each spin-1/2 system act on its own subspace. Students are asked to explain the characteristics of the operators that will be diagonal in the uncoupled representation and they are given multiple opportunities to test what they predict in concrete situations and reconcile the differences between their predictions and observations if there are any. At the end of the first part of the QuILT, students are given the following question to help them understand that the basis vectors can be chosen according to convenience.

- Consider the following conversation between Andy and Caroline and explain with whom you agree. 
Andy: For the question about choosing a basis for two spin-1/2 systems, we do not necessarily have to choose a basis in the product space which consists of eigenstates of $\hat{S}_{1 z}$ and $\hat{S}_{2 z}$.

Caroline: I disagree. We must choose a basis in the product space such that the basis vectors are eigenstates of $\hat{S}_{1 z}$ and $\hat{S}_{2 z}$.

The QuILT helps students learn, e.g., that usually if an operator can be represented by a diagonal matrix in a particular basis, that basis is more convenient than others. It is not necessary to always choose a basis in the product space which is an eigenstate of $\hat{S}_{1 z}$ and $\hat{S}_{2 z}$. Discussion about these questions also leads to a smooth transition to other basis vectors, e.g., from the uncoupled to the coupled representation.

\section{Introducing the coupled representation}

The section of the QuILT that introduces the coupled representation asks students to list all of the possible total spin quantum numbers $s$ for the total spin angular momentum $\vec{S}=\vec{S}_{1}+\vec{S}_{2}$ for the product space of two spin- $1 / 2$ systems. They also list the quantum numbers for the $z$ component of total spin $m_{s}$ when $s=1$ and 0 . Students contemplate why a complete set of coupled states denoted by quantum numbers $s$ and $m_{s}$ and written as $\left|s, m_{s}\right\rangle$ forms a set of basis vectors for the product space for a system of two spin-1/2 systems. Some guided inquiry-based questions help students learn to apply different operators such as $\hat{S}^{2}, \hat{S}_{1}^{2}, \hat{S}_{2}^{2}$, and $\hat{S}_{z}$ on the states $\left|s, m_{s}\right\rangle$. Students also verify that the basis vectors in the coupled representation are orthonormal. As shown in the multiple-choice question below, the QuILT also helps students contemplate the differences between the coupled and uncoupled basis vectors.

- Choose all of the following statements that are correct about the differences between the "coupled" and "uncoupled" representations of the multi-spin system.

(1) Working entirely within the coupled representation, you cannot decompose the product state of a two-spin system into products of states of each individual spin.

(2) Working entirely within the uncoupled representation, you can decompose the product state of a two-spin system into products of states of each individual spin.

(3) The basis vectors in the uncoupled representation are eigenstates of $\hat{S}_{1}^{2}, \hat{S}_{1 z}, \hat{S}_{2}^{2}$, and $\hat{S}_{2 z}$, whereas the basis vectors in the coupled representation are eigenstates of $\hat{S}^{2}, \hat{S}_{1}^{2}, \hat{S}_{2}^{2}$, and $\hat{S}_{z}=\hat{S}_{1 z}+\hat{S}_{2 z}$.

All of the three options in the question above are correct. Through these types of questions, students learn that in the coupled representation the basis vectors in the product space are such that the individual states of the two particles cannot always be separated from each other. However, the basis vectors of the coupled representation can be written as linear combinations of the basis vectors of the uncoupled representation. They also observe that the basis vectors in the coupled and uncoupled representations are not the eigenstates of the same operators. For example, the basis vectors in the coupled representation are the eigenstates of the square of the total spin operator $\hat{S}^{2}$, but the basis vectors in the uncoupled representation are not the eigenstates of this operator. On the other hand, the activities in the QuILT help them learn that the basis vectors in both the coupled and uncoupled representations are eigenstates of the operators $\hat{S}_{1}^{2}$ and $\hat{S}_{2}^{2}$.

\section{Constructing the matrices for various operators in the product space of two spin-1/2 systems in the coupled representation}

In the second part of the QuILT, students are given the task of writing the same operators $\hat{H}_{1}=\gamma\left(\hat{\vec{S}}_{1} \cdot \hat{\vec{S}}_{2}\right)$ and $\hat{H}_{2}=\mu\left(\hat{\vec{S}}_{1} \cdot \vec{B}+\hat{\vec{S}}_{2} \cdot \vec{B}\right)$ in matrix form in the coupled representation that they had earlier learned to write in the uncoupled representation via a guided inquiry process (without using the CGC table). They are asked to compare the matrices in the coupled representation with those in the uncoupled representation. They also learn that in the coupled representation, it is convenient to write the operator $\hat{\vec{S}}_{1} \cdot \hat{\vec{S}}_{2}=\left(\hat{S}^{2}-\hat{S}_{1}^{2}-\hat{S}_{2}^{2}\right) / 2$ so the matrix elements can be easily calculated. There are discussions in the QuILT to help students understand why an operator, e.g., $\hat{\vec{S}}_{1} \cdot \hat{\vec{S}}_{2}$, is diagonal in the coupled basis but nondiagonal in the uncoupled basis. Students also explore and learn that the operator $\hat{H}_{2}=\mu\left(\hat{\vec{S}}_{1} \cdot \vec{B}+\hat{\vec{S}}_{2} \cdot \vec{B}\right)$ is diagonal in both the coupled and uncoupled representations since the basis vectors in the coupled representation are the eigenstates of the operator $\hat{S}_{z}=\hat{S}_{1 z}+\hat{S}_{2 z}$ (since the magnetic field is in the $z$ direction). Then they are asked to express the matrix for $\hat{H}_{2}$ in the block diagonal form where all the nonzero terms are confined to a smaller block rather than being spread out in the full $4 \times 4$ matrix. This process helps students understand that they can arrange the order of basis vectors as they wish while constructing the matrix.

\section{PRELIMINARY EVALUATION}

Based on the investigation of students' difficulties, we designed a pre-test and a post-test to assess the issues related to the addition of angular momentum. The pretest was administered to the entire undergraduate quantum mechanics classes (altogether 26 students) in the 2009 spring semester and 2010 spring semester after traditional instruction. After the pre-test, the same two groups of students worked on the QuILT, and the post-test was 
TABLE I. Correctness percentage of 26 students answering question (a) in the pre-test and post-test. The items represent the types of students' correct or incorrect answers and the corresponding numbers stand for the number of students giving a particular type of answer. The item "mixed" means that the students expressed the coupled singlet and triplet states using the uncoupled basis vectors. The item "dimension" represents the mistakes related to the dimension of the Hilbert space, e.g., only writing down the basis vectors in the subspace of one of the spins.

\begin{tabular}{lccc}
\hline \hline \multicolumn{2}{c}{ Pre-test question (a) } & \multicolumn{2}{c}{ Post-test question (a) } \\
Correct 77\% & Incorrect 23\% & Correct 85\% & Incorrect 15\% \\
\hline Uncoupled 9 & Dimension 4 & Uncoupled 6 & Dimension 4 \\
Coupled 4 & Mixed 2 & Coupled 16 & Mixed 0 \\
Mixed 7 & & Mixed 0 & \\
\hline \hline
\end{tabular}

administered to them the following week in class. The questions in the pre-test and the post-test were similar but used product spaces for quantum systems with different spin. In particular, in the pre-test, the system contained two spin- $1 / 2$ particles, while the system in the post-test had one spin- $1 / 2$ particle and one spin- 1 particle. The pre-test question asks the following.

- Two spin- $1 / 2$ systems (with the spin quantum numbers $s_{1}=1 / 2$ and $s_{2}=1 / 2$ ) at fixed locations in space (only consider spin degrees of freedom) interact with each other, and with a uniform magnetic field $\vec{B}$ pointing in the $+z$ direction. When the magnetic field is off, the interaction between the spins is given by the Hamiltonian

$$
\hat{H}_{1}=\left(4 E_{0} / \hbar\right) \hat{\vec{S}}_{1} \cdot \hat{\vec{S}}_{2}=\left(2 E_{0} / \hbar\right)\left(\hat{S}^{2}-\hat{S}_{1}^{2}-\hat{S}_{2}^{2}\right),
$$

where $\hat{\vec{S}}=\hat{\vec{S}}_{1}+\hat{\vec{S}}_{2}$ and $E_{0}$ is a constant. The magnetic field interacts with each spin as follows:

$$
\hat{H}_{2}=-\mu\left(\hat{\vec{S}}_{1} \cdot \vec{B}+\hat{\vec{S}}_{2} \cdot \vec{B}\right) .
$$

(a) Write down a complete set of basis vectors for the vector space of a system of two spin-1/2 particles. Explain the labels you are using to identify your basis states.

(b) Express the Hamiltonian $\hat{H}_{1}$ in the basis you have chosen. (Write it down as an $N \times N$ matrix).

(c) Express the Hamiltonian $\hat{H}_{2}$ in the basis you have chosen.

(d) Are both $\hat{H}_{1}$ and $\hat{H}_{2}$ diagonal matrices in the basis you chose?

Questions (a), (b), and (c) weigh 3 points each and question (d) weighs 1 point. The correctness percentage of all the 26 students answering question (a) in the pre-test and post-test is listed in Table I. In the pre-test, 9 students chose the uncoupled basis and 4 students wrote the coupled basis vectors correctly. Another 7 students correctly expressed the coupled singlet and triplet states using the uncoupled basis vectors, e.g., $|1,1\rangle=|\uparrow \uparrow\rangle$ and $|0,0\rangle=$ $\frac{1}{\sqrt{2}}(|\downarrow \uparrow\rangle-|\uparrow \downarrow\rangle)$. Other students gave incorrect responses, e.g., $|1 / 2, \pm 1 / 2\rangle_{1}$ and $|1 / 2, \pm 1 / 2\rangle_{2}$, which indicated that they believed that the basis vectors in the product space are the same as the basis vectors in the subspaces. In the posttest, most of the students chose the coupled basis vectors when answering the first question (but for the product space of a spin- $1 / 2$ and a spin-1 system). However, four students did not provide the correct answer because they mistakenly treated the post-test question as a two spin- $1 / 2$ particles system or a three spin- $1 / 2$ particles system.

The correctness percentages of the students answering questions (b) and (c) in the pre-test and post-test are listed in table II. Only 2 out of the 26 students correctly calculated the matrices for both the operators $\hat{H}_{1}$ and $\hat{H}_{2}$ in the pre-test. About $27 \%$ of the students incorrectly calculated the matrices for the operators $\hat{H}_{1}$ and $\hat{H}_{2}$ in the fourdimensional Hilbert space by simply adding the matrices for the spin operators in the two-dimensional subspaces. Other students had no idea about how to calculate the matrix elements of the operators for a given set of basis

TABLE II. Correctness percentages of 26 students answering questions (b) and (c) in the pretest and post-test. The item "sandwich" means that the students calculated the matrix elements by sandwiching the operator with the basis vectors. The item "simply add" represents the mistake of calculating the matrices by simply adding the matrices for the spin operators in different subspaces as though they were in the same vector space. The number corresponding to

\begin{tabular}{|c|c|c|c|c|c|c|c|c|}
\hline \multicolumn{5}{|c|}{ Pre-test questions (b) and (c) } & \multicolumn{4}{|c|}{ Post-test questions (b) and (c) } \\
\hline \multirow{4}{*}{$\mathrm{b}$} & \multirow{4}{*}{$\begin{array}{c}\text { Correct } 8 \% \\
\text { Sandwich }\end{array}$} & \multirow{4}{*}{2} & \multicolumn{2}{|c|}{ Incorrect $92 \%$} & \multicolumn{2}{|c|}{ Correct $54 \%$} & \multicolumn{2}{|c|}{ Incorrect $46 \%$} \\
\hline & & & Sandwich & 0 & Sandwich & 14 & Sandwich & 7 \\
\hline & & & Simply add & 7 & & & Simply add & 0 \\
\hline & & & No Idea & 17 & & & No Idea & 5 \\
\hline & \multirow{4}{*}{$\begin{array}{c}\text { Correct } 8 \% \\
\text { Sandwich }\end{array}$} & \multirow{4}{*}{2} & \multicolumn{2}{|c|}{ Incorrect $92 \%$} & \multicolumn{2}{|c|}{ Correct $73 \%$} & \multicolumn{2}{|c|}{ Incorrect $27 \%$} \\
\hline \multirow{3}{*}{$\mathrm{c}$} & & & Sandwich & 0 & Sandwich & 19 & Sandwich & 2 \\
\hline & & & Simply Add & 7 & & & Simply Add & 0 \\
\hline & & & No Idea & 17 & & & No Idea & 5 \\
\hline
\end{tabular}
each item stands for the number of students giving a particular type of answer. 
vectors. In the post-test, 21 out of the 26 students correctly knew that the matrix elements can be obtained by sandwiching the operator with the corresponding basis vectors. Among these 21 students, $66 \%$ of them correctly calculated the matrices of both $\hat{H}_{1}$ and $\hat{H}_{2}$ using the coupled representation. Some students who selected either the coupled or uncoupled representation had difficulties in calculating the matrix elements, especially for some offdiagonal elements in $\hat{H}_{1}$ in the uncoupled representation.

The correctness percentages of the students answering question (d) in the pre-test and post-test are listed in Table III. As shown in Table III, only 8 out of the 26 students answered question (d) correctly in the pre-test while the other students did not know whether the operator matrices should be diagonal or not in a particular basis. In the post-test, $85 \%$ of the students who had appropriately chosen either the coupled or uncoupled representation in question (a) correctly answered question (d). However, some of the students who answered question (d) correctly were unable to construct the Hamiltonian matrices in the previous questions. The average correctness percentage for all the questions in the pre-test was 40\% in 2009 (with 9 students) and $27 \%$ in 2010 (with 17 students). After the students learned the topic using the QuILT for the addition of angular momentum, their post-test average score increased to $73 \%$ and $72 \%$ in 2009 and 2010, respectively.

We have conducted a Wilcoxon signed-rank test for students' pre-test and post-test total scores, and the result indicated significant improvement in the post-test performance $(z$ value $>3.5)$. We also conducted a two-tailed $t$-test on students' scores for each question and their total scores in the pre- and post-tests. The $p$ values are less than 0.001 for all questions except for question (a) (on which students had performed reasonably well in the pretest). While the post-test scores are statistically significantly better than the pre-test scores ( $p$ value $<0.001$ ), students' understanding can be improved further. One central reason for the low score on the post-test was that many students treated the post-test problem as though it was a product space of two spin- $1 / 2$ systems as opposed to a product of one spin- $1 / 2$ and one spin- 1 system. However, the fact that a greater percentage of students chose the coupled representation in

TABLE III. Correctness percentage of 26 students answering question (d) in the pre-test and post-test. Students who did not provide the correct response either provided wrong answers or left the question blank (which are represented by the items "wrong" and "no answer"). The number corresponding to each item stands for the number of students giving a particular type of answer.

\begin{tabular}{lccc}
\hline \hline \multicolumn{2}{c}{ Pre-test question (d) } & \multicolumn{2}{c}{ Post-test question (d) } \\
\hline Correct 31\% & Incorrect 69\% & Correct 85\% & Incorrect 15\% \\
Right answer 8 & Wrong 9 & Right answer 22 & Wrong 2 \\
& No answer 9 & & No answer 2 \\
\hline \hline
\end{tabular}

the post-test than in the pre-test indicates that, after using the QuILT, the students are aware of the fact that choosing the basis vectors to be the eigenstates of the operators can make the calculations easier. Also, when answering questions (b) and (c) in the post-test, many students who had used the tutorial could construct the diagonal or offdiagonal operator matrix by sandwiching the operator with the basis vectors in the product space.

\section{SUMMARY AND CONCLUSION}

We find that students have many common difficulties related to the addition of angular momentum. For example, many students were unclear about the dimension of the product space and they incorrectly believed that the dimension of the product space is the sum of the dimensions of the subspaces. Students also had difficulty in distinguishing between the basis vectors in the coupled and uncoupled representations and had difficulty in determining how to choose an appropriate basis for the product space to answer questions related to the measurements of different observables. While changing basis using a CGC table, students also had difficulty in determining how to calculate the probabilities of measuring different observables. Students struggled to construct the matrix of an operator in a convenient basis in the product space. Some students believed that the dimension of a product space in the coupled and uncoupled representations is different. In particular, some students simply added the matrices for two spin- $1 / 2$ particles to construct the matrix of the operator $\hat{S}_{1 z}+\hat{S}_{2 z}$ such that the resulting matrix in the product space was still two dimensional in the uncoupled representation. Some students had difficulty understanding why the operator $\hat{S}_{1 z}+\hat{S}_{2 z}$ is diagonal in the coupled representation but $\hat{S}_{1 z}+\frac{1}{2} \hat{S}_{2 z}$ is not. Some believed that they must be given the Hamiltonian of the system in order to write any operator in the matrix form in a given basis.

We developed a research-based QuILT to improve students' understanding of the addition of angular momentum. It provides a guided approach to bridge the gap between the quantitative and conceptual issues related to addition of angular momentum and helps students connect different concepts and build a knowledge structure. The QuILT keeps students actively engaged in the learning process. Our preliminary assessment shows that the QuILT improves students' understanding of concepts related to the addition of angular momentum.

\section{ACKNOWLEDGMENTS}

This material is based upon work supported by the National Science Foundation under Grants No. NSF-PHY 1202909 and No. 0855424. We thank the professors who administered the free-response and multiple-choice questions to their students during the investigation of difficulties and administered the QuILT. 
[1] D. Styer, Common misconceptions regarding quantum mechanics, Am. J. Phys. 64, 31 (1996).

[2] P. Jolly, D. Zollman, S. Rebello, and A. Dimitrova, Visualizing potential energy diagrams, Am. J. Phys. 66, 57 (1998).

[3] I. D. Johnston, K. Crawford, and P. R. Fletcher, Student difficulties in learning quantum mechanics, Int. J. Sci. Educ. 20, 427 (1998).

[4] D. Zollman, S. Rebello, and K. Hogg, Quantum physics for everyone: Hands-on activities integrated with technology, Am. J. Phys. 70, 252 (2002).

[5] M. Wittmann, R. Steinberg, and E. Redish, Investigating student understanding of quantum physics: Spontaneous models of conductivity, Am. J. Phys. 70, 218 (2002); Research on teaching and learning of quantum mechanics, in Proceedings of the National Association for Research in Science Teaching, Boston, MA, 1999, http://perg.phys .ksu.edu/papers/narst/; also Am. J. Phys. 70, 199 (2002), published in conjunction with the Gordon Conference on Physics Research and Education on Quantum Mechanics.

[6] C. Singh, Student understanding of quantum mechanics at the beginning of graduate instruction, Am. J. Phys. 76, 277 (2008); Student understanding of quantum mechanics, Am. J. Phys. 69, 885 (2001);

[7] L.D. Carr and S.B. McKagan, Graduate quantum mechanics reform, Am. J. Phys. 77, 308 (2009).

[8] C. Singh, M. Belloni, and W. Christian, Improving student's understanding of quantum mechanics, Phys. Today 59, No. 8, 43 (2006).

[9] A. J. Mason and C. Singh, Do advanced students learn from their mistakes without explicit intervention?, Am. J. Phys. 78, 760 (2010); A. Mason and C. Singh, Reflection and self-monitoring in quantum mechanics, AIP Conf. Proc. 1179, 197 (2009).

[10] G. Zhu and C. Singh, Surveying students' understanding of quantum mechanics, AIP Conf. Proc. 1289, 301 (2010); C. Singh and G. Zhu, Students' understanding of the addition of angular momentum, AIP Conf. Proc. 1413, 355 (2012); G. Zhu and C. Singh, Students' difficulties with quantum measurement, AIP Conf. Proc. 1413, 387 (2012); C. Singh and G. Zhu, Cognitive issues in learning advanced physics: An example from quantum mechanics, AIP Conf. Proc. 1179, 63 (2009); S. Siddiqui and C. Singh, Surveying instructors' attitudes and approaches to teaching quantum mechanics, AIP Conf. Proc. 1289, 297 (2010).

[11] G. Zhu and C. Singh, Surveying students' understanding of quantum mechanics in one spatial dimension, Am. J. Phys. 80, 252 (2012).

[12] C. Singh, Interactive learning tutorials on quantum mechanics, Am. J. Phys. 76, 400 (2008); Helping students learn quantum mechanics for quantum computing, AIP Conf. Proc. 883, 42 (2007).

[13] G. Zhu and C. Singh, Improving students' understanding of quantum measurement. I. Investigation of difficulties, Phys. Rev. ST Phys. Educ. Res. 8, 010117 (2012); Improving students' understanding of quantum measurement. II. Development of research-based learning tools, Phys. Rev. ST Phys. Educ. Res. 8, 010118 (2012).

[14] G. Zhu, Improving students' understanding of quantum mechanics, Ph.D. thesis, University of Pittsburgh, 2011.

[15] E. Mazur, Peer Instruction: A User's Manual. (PrenticeHall, Upper Saddle River, NJ, 1997).

[16] C. Singh, Transfer of learning in quantum mechanics, AIP Conf. Proc. 790, 23 (2005).

[17] S. Y. Lin and C. Singh, Categorization of quantum mechanics problems by professors and students, Eur. J. Phys. 31, 57 (2010); Assessing expertise in quantum mechanics using categorization task, AIP Conf. Proc. 1179, 185 (2009).

[18] C. Singh, Student difficulties with quantum mechanics formalism, AIP Conf. Proc. 883, 185 (2007).

[19] C. Singh, Assessing and improving student understanding of quantum mechanics, AIP Conf. Proc. 818, 69 (2006).

[20] F. W. Byron and R. W. Fuller, Mathematics of Classical and Quantum Physics (Addison-Wesley, Reading, MA, 1969).

[21] G. Zhu and C. Singh, Improving students' understanding of quantum mechanics via the Stern-Gerlach experiment, Am. J. Phys. 79, 499 (2011); Students' understanding of Stern-Gerlach experiment, AIP Conf. Proc. 1179, 309 (2009). 